TA-KONZEPTE UND -METHODEN

\section{Transgene Nutzpflanzen der 2. Generation - Überlegungen zu einer adäquaten $\mathrm{TA}^{*}$}

\author{
von Stephan Albrecht, Universität Hamburg
}

Wichtige Impulse zur Entwicklung transgener Nutzpflanzen der 2. Generation (trN2) resultieren aus Erfahrungen mit der jedenfalls teilweise fehlgeschlagenen normativen gesellschaftlichen Etablierung ${ }^{1}$ von transgenen Nutzpflanzen mit Merkmalen wie Herbizidtoleranz oder Insektenresistenz. Die analytische Aufarbeitung dieser Probleme ist ein Hintergrund für einen konzeptionellen Rahmen für Technikfolgenforschung und Technikbewertung (TA) zu trN2.

Dieser Rahmen fußt auf vier Säulen: Sicherheits- und Risikoproblemen; Regulierung; Willensbildung und Entscheidungsfindung; sozialen und wirtschaftlichen Probleme. Zu diesen Säulen als thematische Felder für TA werden jeweils zentral wichtige Forschungsfragen benannt.

Der Beitrag soll eine kritische Konzeption von TA fördern und weiterentwickeln, die nicht erst post festum die Scherben des wissenschaftlich-technischen Fortschritts katalogisiert.

\section{Zur Rolle der TA}

Die analytische und kommunikative Aufgabe von TA besteht in der Erweiterung von Entscheidungsgrundlagen, in der Vermehrung von Optionen. Diese Aufgabe erfolgreich wahrzunehmen, ist mit das Schwierigste im Bereich der TA-Forschung überhaupt. Denn hier gilt es, eine Gratwanderung zu organisieren zwischen Affirmation der von den Technikentwicklern propagierten Wege einerseits und einer Opposition gegen eben diese Pläne und Vorhaben andererseits. Gefragt ist aber ein tertium, mit dem größere Klarheit der Prämissen und Folgen der vorgeschlagenen Vorhaben und zugleich möglicher anderer Wege geschaffen wird.
Ein solcher Zuschnitt von TA bedingt, wenn es um Vorhaben in der Forschung geht, eine Offenheit auch der Technikentwickler zur Modifizierung ihrer Ziele und Projekte mittels gewonnener Einsichten. Dabei sind es nicht die TA-Entwickler, die diese Einsichten hätten, sondern es ist der gemeinsame Forschungsund Kommunikationsprozess, der die Möglichkeit einer Weiterentwicklung von Forschungsdesigns, Technologieprojekten und Zielbündeln eröffnet.

Sieht man sich die reichlich verfahrene und häufig von kurzfristiger Taktik bestimmte öffentliche Debatte und Politik um transgene Nutzpflanzen an, so ist ohne neue und weiterentwickelte politische und wissenschaftliche Zugänge der Weg zu einer angemessenen gesellschaftlichen Gestaltung derartiger Innovationen weiterhin nicht abzusehen. Die beteiligten Wissenschaften haben die Chance und auch eine Mitverantwortung, Prozesse zu initiieren und zu betreiben, die neue Grundlagen für gesellschaftliche Entscheidungen mit zu schaffen geeignet sein könnten. Eine TA wie im Folgenden vorgeschlagen könnte $\mathrm{zu}$ einer solchen wichtigen Zukunftsaufgabe in der Gesellschaft bedeutsame Beiträge leisten.

\section{Die Ausgangslage}

Nach in letzter Zeit verbreitetem Bekunden ist die gesellschaftliche Vermittlung der 1. Generation transgener Nutzpflanzen insgesamt nicht gelungen. Wenn schon Robert Shapiro, bis zum Aufkauf durch Pharmacia Upjohn Ende 2000 Vorstandsvorsitzender von Monsanto, ein Misslingen der normativen Etablierung der herbizidtoleranten, teils insektenresistenten transgenen Nutzpflanzen öffentlich konstatiert, so wird man nicht viele weitere Belege anführen müssen. Mittlerweile ist auch bei der internationalen Marktsituation eine für transgene Produkte ambivalente Entwicklung festzustellen. In den USA weigern sich Agrarhandelshäuser zunehmend, transgene Produkte aufzukaufen. Dies führte im Jahr 2001 gegenüber dem Vorjahr zu Rückgängen in der Anbaufläche von transgenem Soja (ca. 9\%), Baumwolle (ca. 12\%) und Mais (ca. $24 \%$ ). Eine Imagekampagne für 50 Mio. US\$ sollte hier gegensteuern. Die führenden Handelshäuser in Großbritannien verlangen von ihren Fleischlieferanten die Zusage, dass in der 
Tierfütterung kein transgenes Material Verwendung findet.

Relevante europäische Nahrungsmittelhersteller verlangen von ihren Zulieferern die $\mathrm{Zu}$ sage, dass die verwendeten Rohmaterialien frei von transgenen Organismen sind. Außereuropäische Staaten wie Japan, Mexiko, Korea, Thailand und Brasilien haben strenge Kennzeichnungspflichten statuiert ${ }^{2}$.

Das mögliche Misslingen einer breiten Einführung von transgenen Nutzpflanzen der 1. Generation wirft die Frage auf, welche Gründe hierfür verantwortlich sind. Auch wenn es dazu keine übereinstimmende Antwort gibt, so werden doch etliche Aspekte vielfach kongruent vorgetragen:

- Die agronomischen Vorzüge insbesondere der Herbizidtoleranz, aber auch der (partiellen) Insektenresistenz, sind nur für die Landwirte von Bedeutung, nicht aber für die Konsumenten der so produzierten Getreide, Baumwolle u. a. Rohstoffe. Es fehlt demnach an der sonst häufigen Interessentenkoalition von Verbrauchern und Produzenten, nach der Gleichung Produktivitätsfortschritt $=$ niedrigerer Preis.

- Die betriebswirtschaftlich positiven Effekte des Einsatzes transgener Sorten sind je nach geographischen und meteorologischen Bedingungen recht unterschiedlich ausgeprägt, teilweise verschwinden sie sogar ganz; Gleiches gilt für die Wirksamkeit der Breitbandherbizide.

- Durch die Kombination der herbizidtoleranten transgenen Sorten mit den passenden Herbiziden ist eine Abhängigkeit der Landwirte von einem oder zwei Herbiziden und damit zugleich von wenigen Agrarchemiefirmen gegeben. Starke Abhängigkeiten bergen Risiken.

- Die wesentlichen Eigenschaften der transgenen Sorten der 1. Generation haben keinen substantiellen Bezug zu dem propagierten Ziel der Sicherung und Verbesserung der Welternährungslage. Denn es sind diejenigen Nutzpflanzen gentechnisch verändert worden, die für die Märkte in den gemäßigten Zonen bedeutsam sind und nicht die für die Subsistenzlandwirtschaft relevanten Nutzpflanzenarten. Entsprechendes gilt für die Eigenschaften, die verändert oder eingefügt worden sind.
- Unabhängig davon, als wie weit gehend man ökologische Folgeprobleme des Einsatzes der transgenen Sorten einschätzt, ist von den Technikentwicklern und -vermarktern die Vielfalt und Komplexität von möglichen oder denkbaren Implikationen unterschätzt worden ${ }^{3}$.

- Die wissenschaftliche und allgemeine Kommunikation ist seitens der interessierten Firmen und Wissenschaftler nach weitest gehend untauglichen bis kontraproduktiven Mustern betrieben worden ${ }^{4}$.

- Die ethischen und moralischen Dimensionen (,Fummelt nicht an unseren Nahrungsmitteln herum!") werden von den Proponenten krass unterschätzt; so auch das klare Ergebnis (ca. $70 \%$ ) des Eurobarometer's (2001).

- TA hat in den Kontroversen um herbizidtolerante und andere transgene Nutzpflanzen eine eher marginale Rolle gespielt. Das dürfte zu aller erst darin begründet liegen, dass es TA-Studien im Lehrbuchsinne zu diesem Themenfeld gar nicht gegeben hat; vielmehr gibt es einen Flickenteppich von Studien und Arbeiten zu Einzelaspekten ${ }^{5}$. Wobei die Teilstudien, die sich mit ökologischen Fragekomplexen befasst haben, einen wichtigen Anteil daran hatten, dass offene Fragen als offene in Erinnerung geblieben sind, weiterführende und differenzierende Fragestellungen aufgeworfen wurden ${ }^{6}$. TA hat sich auf diese Weise eher als Fragengenerator denn als Antwortgeber in dem oben zitierten Lehrbuchsinn erwiesen?

Autoritativ intendierte Versuche, einen definitiven Stand des Wissens als Referenz für weitere Debatten zu etablieren, sind in dieser Intention fehlgegangen, so das Verfahren beim Wissenschaftszentrum Berlin $1991 \mathrm{ff}^{8}{ }^{8}$ Social impact assessment-Studien, d. h. Arbeiten zu den sozial-ökonomischen und sozial-ökologischen Fragenkreisen, als konzeptionell gleich gewichtiger Teil von TA sind quantitativ ungleich weniger durchgeführt worden ${ }^{9}$; insbesondere sind die wichtigen langfristig orientierten Arbeiten aus den Enquete-Kommissionen des Deutschen Bundestages zur Technikfolgenabschätzung in der zweiten Hälfte der 80er Jahre nicht weiterverfolgt worden.

Alle Debatten um transgene Nutzpflanzen der 2. Generation $(\operatorname{trN} 2)$ finden nun u. a. vor diesem knapp skizzierten Hintergrund statt. Ich 
versuche im Folgenden, einen konzeptionellen Rahmen für TA-Forschungen und TAKommunikation im Zusammenhang mit der Entwicklung von trN2 zu umreißen. Dabei unterscheide ich zunächst nicht nach Perspektive oder institutioneller Zuordnung der hier vorgeschlagenen TA; diese Konkretisierungen sollten sich idealiter im Laufe der von mir angestrebten Diskussion herausbilden ${ }^{10}$. Jede derartige Konzeption aber sollte, so meine Prämisse, Erfahrungen aus den Kontroversen, Monologen und Fehlschlägen der vergangenen 15 Jahre einbeziehen. Zugleich ist es schier unumgänglich, die gegenwärtigen Krisensymptome (kulturelle und wirtschaftliche Marginalisierung des ländlichen Raumes, BSE, MKS etc.) und deren tiefer liegende Ursachen und Zusammenhänge in der Landwirtschaft und dem Ernährungsgewerbe in Europa wie global $\mathrm{zu}$ berücksichtigen. Dies nicht etwa nur als Faktor aktueller öffentlicher Meinungen, sondern auch und vor allem als Anfrage an die Verantwortung von Wissenschaften, zum Gemeinwohl i. S. der Menschen- und Grundrechte beizutragen.

\section{Die Säulen der TA zu trN2}

Nach den Plänen der Technikentwickler und Biotechnologen sind trN2 u. a. durch folgende Merkmale charakterisiert: sie sollen

- einen unmittelbaren Nutzen für Endverbraucher aufweisen;

- durch gezielte Veränderung von Pflanzeninhaltsstoffen einen ernährungsphysiologischen Vorteil i. S. eines vorbeugenden Gesundheitsschutzes beinhalten (,functional food", ,health food");

- die Synthese von medizinisch relevanten Proteinen realisieren;

- für die Industrie nachwachsende Rohstoffe (NR) verfügbar machen ${ }^{11}$.

Ein solches Profil zukünftiger Nutzpflanzengenerationen ${ }^{12}$ ist, wie sogleich zu sehen ist, nicht insgesamt neu. Insbesondere das zuletzt genannte Profilelement gehörte auch bislang schon zum programmatischen Repertoire. Die neu hinzugekommenen Elemente sind indessen gewichtig genug, so dass ich mich darauf konzentriere.
TA sollte thematisch auf vier Säulen aufbauen (s. Abb. 1):

- Sicherheits- und Risikofragen,

- Regulierung,

- Entscheidungs- und Willensbildung,

- soziale und wirtschaftliche Implikationen.

\section{Abb. 1: Die Säulen der TA zu trN2}

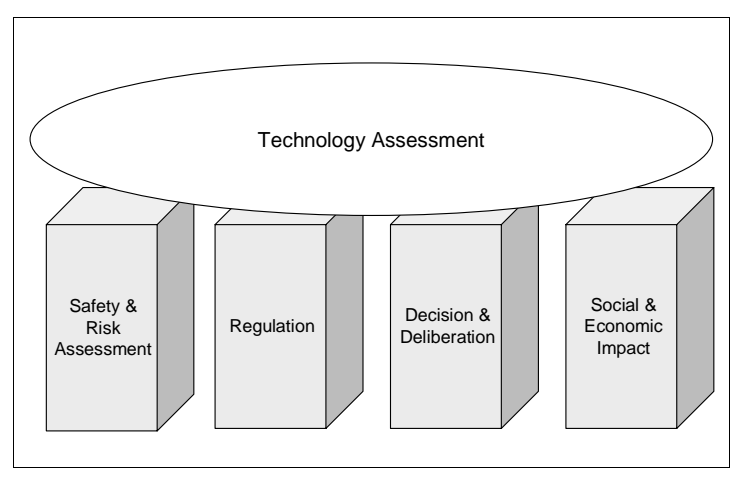

Sicherheits- und Risikofragen betreffen sowohl außermenschliche ökologische Zusammenhänge wie auch die menschliche Gesundheit. Die anvisierten Ziele der Bereitstellung von „functional food" oder von Pflanzen, die medizinisch relevante Substanzen produzieren, zeigen, dass hier erheblicher Forschungsbedarf absehbar ist. Diese Forschungen können sich auf Produkte, seien dies Pflanzen oder einzelne Inhaltsstoffe, beziehen, wie üblicherweise vorgegangen wird. Wenn allerdings, wie z. B. in dem Programm topPlantnord ${ }^{13}$, nicht einzelne Produkte, sondern eine Technologieplattform angestrebt wird, so tut TA gut daran, sich auch mit den Sicherheits- und Risikofragen auf der Ebene der Technologieplattform bzw. einzelnen ihrer Teile auseinander zu setzen.

Dabei könnte prinzipiell jeweils in Bezug auf die einzelnen Technologien und auch ihr Zusammenwirken gefragt werden:

- Welche Wirkungen sind möglich?

- Welche Risiken sind denkbar?

- Welche Elemente der Technologie generieren u. U. welche Risiken?

- Welche Möglichkeiten der Minderung oder Eliminierung gibt es?

- Welche Elemente könnten oder sollten daher entwickelt werden?

Weiterführend in diesem Zusammenhang könnte die Definition eines „Qualitätsstan- 
dards" sein, der sich ergäbe aus Kriterien- und Indiaktorenkatalogen. Er würde ausgehen von der oben skizzierten Prüfliste und die Qualitäten von verfügbaren Techniken und Methoden beschreiben mitsamt der bestehenden Unsicherheiten. Grundlage eines solchen Vorgehens wäre ein umfassender kritischer Review von Techniken und Methoden, wie sie z. B. Ingo Potrykus (1991) vorgelegt hat ${ }^{14}$. Auf einer solchen Basis ließen sich dann Merkmale kombinieren, die eine Qualifizierung von Methodiken und Techniken erlaubten. Eine solche Qualifizierung und Standardisierung wäre zugleich ein wichtiger Beitrag zur Transparenz der Forschung und Entwicklung.

Das Beispiel von Antibiotikaresistenzgenen als Marker in transgenen Nutzpflanzen hat aus meiner Perspektive gezeigt, wie es eher nicht gehen sollte. Hier ist aus Praktikabilitätsgründen viel Zeit verloren worden.

Heute wäre es vorteilhaft, wenn man über praxistaugliche alternative Verfahrensweisen verfügen könnte. Es hat an ernsthaften Stimmen schon seit geraumer Zeit nicht gefehlt, Antibiotikaresistenzen als Marker aus Gründen der Vorsorge nicht mehr zu verwenden. Auch wenn naturwissenschaftlich und medizinisch längst nicht definitiv geklärt ist, welche Risiken wie realistisch sind, so hat sich doch die Auffassung durchgesetzt, von Markern mit Antibiotikaresistenzgenen ganz abzugehen ${ }^{15}$. Die Etablierung eines Qualitätsmaßstabes für Risiko- und Sicherheitsaspekte könnte neben der positiven Wirkung nach außen zugleich nach innen erheblich dazu beitragen, dass in Zukunft sowohl systematisch wie frühzeitig etwaige Probleme gesucht und beobachtet werden könnten.

Die angestrebten Merkmale der trN2 bedingen zugleich zwei Weiterungen der Sicherheits- und Risikofragen. Bisherige Debatten um ,novel food“ haben das Konzept der „substantial equivalence“, einen Vergleich konventioneller mit neuartigen Lebensmitteln herangezogen. Dieses Konzept, Anfang der 90er Jahre in der OECD entwickelt, ist der naturwissenschaftliche Versuch, eine Brücke zwischen den Paradigmenwelten der Erfahrung und des Experimentierens zu bauen, aus Übereinstimmungen bzw. aus fehlenden oder nicht erkennbaren Abweichungen auf Gleichartigkeit zu schließen. Solche Methodik ist umstritten, was nicht verwunderlich ist, da der Gegenstand, an dem sich die Methodik entwickelt hat, transgene Nahrungsmittel sind. Die Royal Society of Canada (RSC) hat das Konzept der „substantial equivalence“ als ungeeignet für die Beurteilung von transgenen Nahrungsmitteln qualifiziert $^{16}$. Unabhängig davon, ob man sich die Kritik und Vorschläge der RSC zu eigen macht, bleibt als wichtig die Erkenntnis festzuhalten, dass qua definitione für trN2 die spezifische Neuartigkeit als Charakteristikum gilt.

Mit der Herstellung von trN2 wird ein weiterer Schritt getan in dem schon seit einigen Jahren zu beobachtenden fundamentalen Umschwung im Lebensmittelbereich, nämlich einem Paradigmenwechsel von der Erfahrung zum Experiment ${ }^{17}$. Mir scheint von daher offensichtlich, dass weit reichende methodische Innovationen in der Prüfung und Zulassung von neuartigen Nahrungsmitteln erforderlich sind, die auch auf die spezifischen Methoden rekurrieren, mittels derer diese Nahrungsmittel konstruiert und produziert worden sind. Dabei könnte auf die Systematik aufgebaut werden, die die RSC für transgene Nahrungsmittel der 1. Generation entwickelt und vorgeschlagen hat. Man könnte auch aus den Problemen im Kontext von BSE zusätzliche Argumente entnehmen für die Bedeutung von Prozessen der Nahrungsmittelherstellung: „Process matters, not only content ${ }^{\text {‘18 }}$.

Hier sehen wir die erste Ausweitung von Sicherheits- und Risikofragen im Zusammenhang mit trN2, nämlich das Erfordernis einer weitgehend neuen Methodik zur Prüfung und Sicherheitsbewertung von Nahrungsmitteln mit neuartigen Eigenschaften. Auf diesem Weg stehen Wissenschaft und zuständige Behörden sehr am Anfang. Es ist nicht nur aus Sicherheitsgründen, sondern auch aus gesellschaftspolitischen Gründen unerlässlich, dass hier nicht eine weitere zeitliche und sachliche Schieflage zwischen Herstellung neuartiger Produkte und Wissen um deren Implikationen hingenommen oder befördert wird.

Die zweite Weiterung geht in den medizinischen Sektor. Wenn Pflanzen medizinisch relevante Proteine oder andere Substanzen synthetisieren sollen, dann sind schon in der Entwicklung dieser Pflanzen, also auch in der Entwicklung darauf orientierter Techniken oder Technologien, die zu pharmazeutischen Ent- 
wicklungen gehörenden Sicherheits- und Risikofragen aufzuwerfen.

Das kompliziert auf eine Weise diese Vorhaben, ist aber eine unabdingbare Voraussetzung dafür, dass nicht erst später bei konkreten Produktentwicklungen alle möglichen Probleme offenbar werden, mit den aus der 1. Generation transgener Pflanzen bekannten nachteiligen Konsequenzen.

Hier wird im Vorgehen sowohl danach zu unterscheiden sein, welcher Art die pharmazeutischen Substanzen sind, die in trN2 synthetisiert werden sollen, wie auch, auf welche Weise das Pharmakon aus der Pflanze gewonnen und (weiter) prozessiert wird.

Schließlich werden auch Aspekte der Sicherheit der Anbauflächen und deren Umgebung zu bedenken sein, wenn pharmazeutisch wirksame Stoffe, die bislang in geschlossenen Anlagen und Fabriken produziert worden sind, nun im offenen Feld angebaut werden. Hinzu kommt, dass der ganze komplizierte Fragenkreis der Regulierung pflanzlicher Substanzen geöffnet wird, soweit von trN2 Pharmazeutika als Pflanzenteil angewendet werden sollen. Bislang sind pharmazeutische Stoffe pflanzlicher Herkunft historisch unsystematisch teils wie andere Arzneimittel auch reguliert, teils nicht nach diesen Regularien zugelassen. Diese Inkonsistenzen der Prüfung und Zulassung werden an Hand von entsprechenden trN2 unweigerlich auf die Agenda geraten.

Es ist von Gegenständen und Methoden her evident, dass dieser Teil der TA von naturwissenschaftlich Ausgebildeten (das schließt die Ernährungs- und Medizinwissenschaften ein) geleistet werden muss. Wobei aus meiner Sicht zentral wichtig ist, die Formulierung der Qualitätsstandards als gemeinsame Arbeit der Technikentwickler und der TA-Entwickler zu gestalten. Da es sich hier um eine Art Pioniervorhaben handelt, ist kein a priori-Rezept anzugeben, wie derartige gemeinsame Formulierungen gelingen können. Handwerkszeug gibt es dazu aus anderen komplexen Zielfindungsund Verständigungsabläufen durchaus ${ }^{19}$.

\section{Regulierungsfragen für trN2}

Regulierungsfragen sind nolens volens eines der ständigen Gebiete von TA zur modernen Biotechnologie. In Bezug auf trN2 ist vor dem
Hintergrund der Erfahrungen vor allem bedeutsam, einen analytischen und konzeptionellen Beitrag zur Entwicklung angemessener Regulierungen zu formulieren. Angemessen heißt in diesem Fall: den verwendeten Prozessen und Produkten und deren Wirkungspotentialen entsprechend.

Die teilweise unübersichtliche Regulierungslandschaft $\mathrm{zu}$ transgenen Nutzpflanzen und ,novel food“" ${ }^{“ 20}$ zeichnet sich durch diverse Defizite aus, die teils struktureller, teils kognitiver und teils prozeduraler Natur sind. Wichtige Defizite sind u. a.:

- horizontale und vertikale Regulierungen interferieren;

- unterschiedliche Traditionsstränge rechtlicher Normierungen sind nur schwer zu integrieren;

- Elemente von Überbürokratisierung finden sich neben bedeutsamen Lücken;

- für wichtige Regulierungskomplexe fehlen unstrittige und/oder unzweideutige wissenschaftliche und methodische Grundlagen;

- gleichwohl bestehen hohe Abhängigkeiten bei und in Regulierungsentscheidungen von wissenschaftlichen Expertengremien oder administrativ-wissenschaftlichen Hybridgremien.

Es ist ein oft zu beobachtendes Spiel, dass Interessenten im Land A auf die angeblich viel sachbezogenere Regulierung im Land B verweisen. Regelmäßig tun Interessenten aus dem Land B das Gleiche mit Hinweis auf Land A. Untersuchungen, insbesondere zum Vergleich zwischen den USA und der EU, zeigen allerdings, dass es keinen Königsweg der Regulierung der modernen Biotechnologie gibt. So wird es auch in Bezug auf trN2 bleiben.

Ein neues Spezifikum der Regulierung wird sich ergeben durch das Hinzutreten medizinisch wirksamer Substanzen. Mit diesem Entwicklungsziel gerät man, wie schon erwähnt, unweigerlich in eine Überschneidungszone mit den Regularien der Prüfung und $\mathrm{Zu}$ lassung von Arzneimitteln. Für trN2 wird es also zumeist eine zweistufige Regulierung geben, nämlich die für Freisetzen und Inverkehrbringen transgener Organismen und die für den jeweiligen weiteren Verwendungszweck (s. Abb. 2) ${ }^{21}$. 
Abb. 2: Regulierungen für $\operatorname{trN2}$

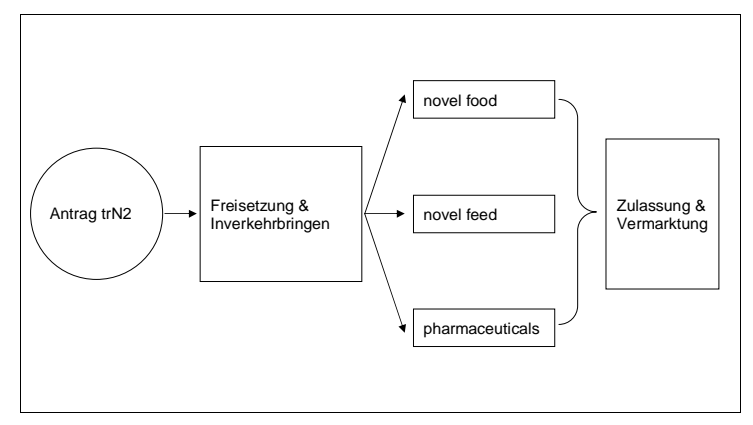

Die von mir in Abschnitt 3 vorgeschlagene Entwicklung eines Qualitätsstandards bei der technologischen Entwicklung und der Konstruktion von trN2 hätte im Erfolgsfalle positive Auswirkungen auch auf die Regulierungsstrukturen und -prozeduren.

Fragestellungen für TA-Untersuchungen im Feld von Regulierungen können insbesondere solche sein, die sich auf die Analyse unterschiedlicher Gegenstandsbereiche von Regulierungen beziehen wie Saatgut, Futtermittel, Pharmaka, Nahrungsmittel, deren Interdependenzen und Interferenzen samt der den Regulierungen zugrunde liegenden Wissensstrukturen (,scientific basis of regulation“) und implizite oder explizite Wertvorstellungen. Ein zweiter Teil von TA-Fragestellungen richtet sich auf die Analyse verschiedener Ebenen und Modi von Regulierungen und deren jeweilige Bedeutungen (räumliche Ebenen; rechtssystematische und -technische Ebenen). Schließlich gehört als ein drittes wichtiges Element dazu die kritische, auch komparative Evaluation bisheriger Regulierungen mitsamt ihrer Implementationen. Dabei geht es um Fragen von Effizienz und Effektivität für unterschiedene Bereiche wie Wissenschaften, Landwirtschaft, Handel, Verbraucherschutz ebenso wie um die Wirkungen von Regulierungen auf die gesellschaftlichen Kontroversen.

\section{Entscheidungs- und Willensbildungsver- fahren bei trN2}

Entscheidungs- und Willensbildungsverfahren sind für Erfolg oder Misserfolg von technologischen Innovationen grundlegend bedeutsam. Die TA-Forschung und -Praxis verfügt international in diesem Feld über einen beachtlichen Erkenntnis- und Erfahrungsfundus ${ }^{22}$. Dabei geht es um Entscheidungs- und Willensbildungsverfahren auf ganz unterschiedlichen Ebenen, von den Wissenschaften bis zu den gesellschaftlichen und politischen Institutionen und Öffentlichkeiten. Für trN2 sind diese Ebenen in Abbildung 3 aufgeführt.

Auf allen diesen Ebenen finden ständig Willensbildungen und Entscheidungen statt, die sich teilweise positiv aufeinander beziehen, teils konträr verlaufen, teils unkoordiniert stattfinden. TA zu trN2 in diesem Feld hat zur Aufgabe, die Analyse der Strukturen und Inhalte von ablaufenden Willensbildungs- und Entscheidungsprozessen zu verbinden mit der Offenlegung der zugrunde liegenden Wert- und Interessenpräferenzen und der experimentellen Etablierung innovativer Formen von Willensbildung und Entscheidung, die den erkannten bisherigen Defiziten ${ }^{23}$ abzuhelfen geeignet sein könnten und die helfen könnten, die Förderung als sinnvoll erkannter technologischer Innovationen als eine gesellschaftliche Gestaltungsaufgabe wahrzunehmen ${ }^{24}$.

\section{Abb. 3: Orte von Willensbildungen und Ent- scheidungen}

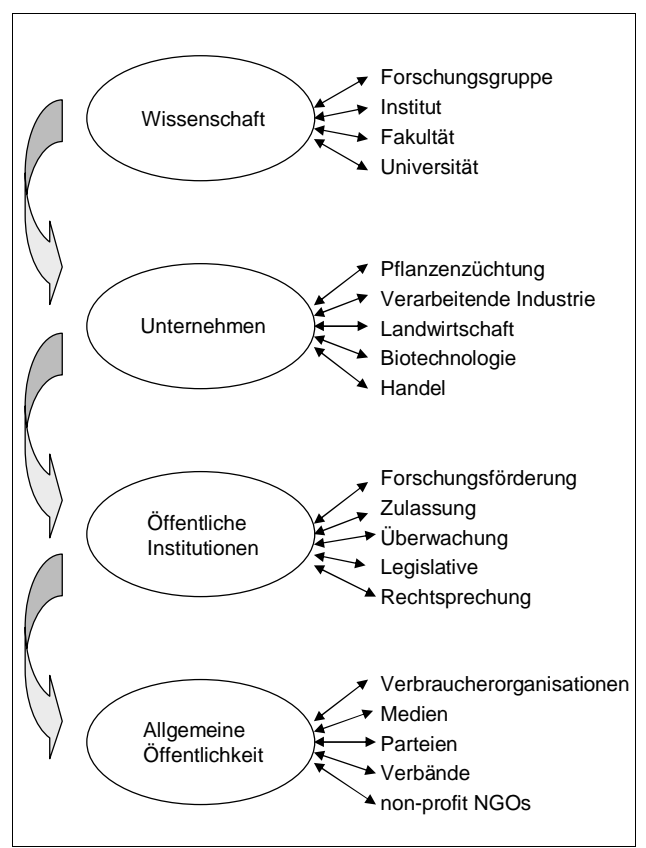

In Bezug auf trN2 sei das beispielhaft skizziert. Das vielfach in Forschungsanträgen und -programmen beschriebene Ziel der Entwicklung von trN2 als ,functional food" ist eine normative Fixierung, nicht eine aus naturwis- 
senschaftlicher Analyse oder Erkenntnis gewonnene Ableitung ${ }^{25}$. In diese normative Festlegung gehen industriewirtschaftliche, gesundheits- und ernährungspolitische, landwirtschaftliche und forschungsstrategische Überlegungen ein. In dem Konzept von topPlantnord z. B. wird die primär zu entwickelnde Technologieplattform als Mittel zum längerfristigen Zweck eines ,wertvollen Beitrag(es) zu einer innovativen und verbraucherorientierten Pflanzenproduktion" gesehen.

Ähnliche Begründungen finden sich auch in thematisch verwandten Projektkonzepten für

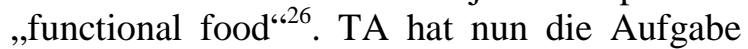
der Offenlegung der in diese Zielformulierung eingegangenen Wertpräferenzen einschließlich der darin enthaltenen Wertkonflikte.

Um einen solchen impliziten Konflikt zu nennen: „Functional food“ als Konzept zur Verbesserung der Ernährungs- und Gesundheitssituation in Deutschland und anderen Industriestaaten wird von unterschiedlichen Akteuren sehr divergent bewertet. Während die Prämisse der zitierten Argumentation der Technikentwickler ist, dass eine solche Strategie sinnvoll ist, reicht die Palette der Aussagen im ernährungswissenschaftlichen Bereich von „Nischenstrategie“ bis zu „funktionelle Lebensmittel sind bestenfalls immer nur (eins von vielen anderen) Hilfsmittel(n), gesund zu leben, das Leben zu genießen, sich wohl zu fühlen“27. Im Bereich von Gesundheits- oder Verbraucherorganisationen ließen sich viele weitere Schattierungen aufzeigen ${ }^{28}$.

Das Offenlegen von Präferenzen betrifft auch Institutionen, die auf die Technologieentwicklung Einfluss ausüben, seien es Ministerien, Krankenkassen, Industrieunternehmen, Interessenverbände oder wer sonst als Akteur oder Beteiligter betroffen ist, wie in Abbildung 3 skizziert.

Hier gibt es etliche richtungweisende Lehren aus den Fehlschlägen und Fehlentwicklungen bei den ersten transgenen Nutzpflanzen. Als einen Fall von ,functional food“ kann man auch den transgenen Reis von Potrykus \& Beyer ansehen ${ }^{29}$. Der darüber öffentlich ausgebrochene Streit ist konkret veranlasst durch großsprecherische und unseriöse Verlautbarungen der Firma Monsanto. Diese überzogenen Proklamationen reproduzieren exakt ein altes Kommunikationsmuster, das Robert Shapiro, wie oben schon erwähnt, öffentlich selbstkritisch als kontraintentional wirkend bewertet hat. Aber auch jenseits dieses Problems stellen sich etliche Fragen, die gegenwärtig, wo erst einige kg Saatgut aus dem Labor dem internationalen Reis-Forschungsinstitut (IRRI) in Manila zur Verfügung gestellt worden sind, nicht beantwortet werden können, die aber behandlungsbedürftig sind; wie z. B. die nach der tatsächlichen quantitativen Verfügbarkeit von Provitamin A aus dem transgenen Reis und die jeweiligen gesundheitlichen Wirkungen der verfügbaren Menge oder auch die nach parallelen oder alternativen Wegen der Ernährungsund Gesundheitssicherung vor allem für Kinder in den Gebieten mit Mangelernährung und Hunger $^{30}$. Hierzu gehört auch die Ursachenanalyse des Vitamin A-Mangels in den betroffenen Gebieten, insbesondere die Zerstörung traditioneller Ernährungssicherungsnetze und die veränderte Inhaltszusammensetzung moderner Nutzpflanzen im Vergleich $\mathrm{zu}$ den traditionellen Sorten. In dieses Debattenfeld gehört schließlich der Entstehungskontext des Potrykus-Projektes. Denn dieses Projekt ist hervorgegangen u. a. aus der Feststellung, dass der mainstream der universitären Biotechnologie sich just nicht um Ernährungsfragen der nicht industrialisierten Länder bekümmert hat, desgleichen auch die großen Wirtschaftsunternehmen.

An diesen Beispielen sieht man den flieBenden Übergang von den Fragen um Willensbildungen und Entscheidungen herum zur vierten Säule, nämlich den sozialen und ökonomischen Fragen. Die Hinzunahme sozialer Kontexte zu Fragen technologischer Innovationen ist mithin nicht etwas von außen, sozusagen politisch Gesetztes, sondern resultiert aus dem wissenschaftlich-gesellschaftlichen Charakter der Forschungen selbst. In diesem Bereich von TAForschung stellen sich die neu-alten Fragen nach Landnutzung, sozialen und betrieblichen Strukturen, die Entwicklung ländlicher Räume, die Veränderung internationaler Beziehungen und Strukturen. Gewichtig sind auch Fragen nach der gesellschaftlichen Organisation von Ernährung und dem Zuschnitt der Ernährungspolitik. Damit ist der mittelgroße Strauß landwirtschafts- und ernährungspolitischer Kontroversen angesprochen. 


\section{Resümee}

Zusammenfassend kann festgestellt werden, dass die Entwicklung von trN2 Fragen aufwirft, die über diejenigen, die aus der Kontroverse um die Transgene der 1 . Generation bekannt sind, noch hinausgehen. Jetzt wird die Beeinflussung von Ernährung und Gesundheit nicht als möglicher Nebeneffekt gesehen, sondern als wichtiger und eigentlicher Zweck. Jede TA, die sich als mitgestaltend versteht, muss auf diese Erweiterung des Themen- und Problemspektrums eingehen. Augenmerk sollte innovative TA gewichtig auch darauf legen, in frühen Entwicklungsstadien neuer Techniken oder Technikplattformen Verständigungsprozesse mit den Biotechnologen zu suchen, um gemeinsam sachliche und prozedurale Konsequenzen aus den Fehlern der Vergangenheit zu ziehen.

* Ich danke Rolf Meyer, Büro für TechnikfolgenAbschätzung beim Deutschen Bundestag (TAB), Berlin, ganz herzlich für kritische Kommentierungen, die auf wesentliche Schwachstellen des ursprünglichen Textentwurfes hingewiesen haben. Desgleichen den Kolleginnen und Kollegen der Pflanzenzüchtungsfirma KWS Saat AG, die einen praxisgeübten Blick auf den Text geworfen haben. Für Schwächen bleibe allerdings nach wie vor ich verantwortlich.

\section{Anmerkungen}

1) Unter „,normativer Etablierung“ wird hier der gesellschaftliche Prozess verstanden, in dessen Verlauf technologische Innovationen als mit den vorherrschenden Werten vereinbar oder für diese Werte förderlich angenommen werden. Normative Etablierung meint insoweit etwas Umfassenderes als der zumeist verwendete Terminus „Akzeptanz“, mit dem überwiegend eine Aufnahme als käufliches Gut in den Warenpool einer Gesellschaft beschrieben wird, vgl. auch Albrecht 1995

2) KWS Saat AG (Einbeck) 2001, pers. Komm.

3) Dass Ereignisketten auftreten, die zunächst als komplett irreal eingeschätzt werden, ist immer wieder zu beobachten, nicht nur in der Evolutionsbiologie und -geschichte.

4) Ausnahmen wie in Deutschland die KWS Saat AG (Einbeck) bestätigen diese Regel. Besonders unrühmlich hat sich die US-amerikanische Firma Monsanto hervorgetan, vgl. dazu z. B. Mon- biot 2000; Lappé, Bailey 1999. Hauptpunkte der verfehlten Kommunikation waren: Es wurde prätendiert, über alle relevanten Informationen zu verfügen, alle praktischen Situationen und Konstellationen „im Griff“ zuhaben. Öffentliche wie wissenschaftliche Einwände und oft auch diejenigen, die solche vorgebracht haben, wurden als sachunkundig, längst widerlegt, abwegig oder aus niederen Beweggründen, vor allem politischen, handelnd abgewiesen. Erwiesen sich Einwände und abweichende Problemsichten als jedenfalls teilweise zutreffend, wurde wiederum so reagiert, als ob es sich um längst bekannte Sachverhalte handelte.

5) So verzeichnet die Datenbank des ITAS/Karlsruhe für den Zeitraum 1986 bis 1998 zu transgenen Nutzpflanzen 39 TA-einschlägige Veröffentlichungseinträge, die überwiegend mit den 28 registrierten Forschungsprojekten zusammenhängen. Gewiss erfassen diese Einträge die thematisch relevanten Publikationen nicht vollständig; darauf kommt es hier auch nicht an. Vielmehr unterstreichen die thematischen $\mathrm{Zu}$ schnitte der Projekte wie der Publikationen die hier getroffene Aussage.

6) Vgl. grundlegend Tiedje et al. 1989, neuerdings z. B. Kjellsson et al. 1994; 1997; Ammann et al. 1999; Schütte et al. 1998; Schütte et al. 2001

7) Das soll keineswegs eine Wertminderung der TA bezeichnen. Wohl ist damit aber eine Wirkungsgrenze der TA angedeutet. Vgl. zur Rolle von TA in der Biotechnologie Albrecht 1997 und als eher formalen Überblick, der auf Landwirtschaft allerdings gar nicht eingeht; Simonis, Droz 1999

8) Vgl. dazu z. B. Dorothea Jansen 2000

9) Immer wieder sind allerdings Marktabschätzungen für transgene Nutzpflanzen und andere biotechnische Produkte vorgenommen worden; diese rechne ich hier nicht zu den SIA-Studien. Der Abstand zwischen Abschätzung der Marktentwicklung und tatsächlicher Marktentwicklung ist über die Jahre etwa gleich groß geblieben. International wiederholt ist die Rolle großer agrarchemischer Unternehmen behandelt worden, auch in Bezug auf die Entwicklung in nicht industrialisierten Ländern (niL), vgl. z. B. Lappé, Bailey 1999; Hobbelink 1989; Hobbelink 1991

10) Rolf Meyer unterscheidet in diesem Zusammenhang zwischen (1) wissenschaftlicher TA, (2) politikberatender TA und (3) einem Netzwerk zwischen Wissenschaft, Industrie, Politik und Gesellschaft, Meyer 2001 (pers. Komm.)

11) Vgl. für die aufgeführten Ziele z. B. topPlantnord 2001; ähnliche Zielbündel ließen sich aus vielerlei Vorhaben anführen. Vgl. dazu als aktuellen Überblick Rögener 2001 
12)Bisweilen sind auch Zielvorstellungen zu lesen wie z. B. die Übertragung von Pilzresistenzen, Stickstofffixierung o. Ä. Diese sind allerdings wegen ihrer enormen technologischen und züchterischen Komplexität wohl eher etwas für die 3. oder 4. Generation transgener Nutzpflanzen. Wobei sich hier schon Fragen nach möglichen oder wünschenswerten Prioritätensetzungen von Forschung und Züchtung stellen.

13)TopPlantnord 2001; www.top-plant-nord.de

14)Derartige Reviews müssen in angemessenen Zeitabständen wiederholt werden. In der zitierten Arbeit von Potrykus hatte dieser bspw. die particle gun-Methode als weitgehend irrelevant eingeschätzt - eine aus heutiger Sicht krasse Fehleinschätzung.

15)Dabei haben Entwicklungen, die mit der Biotechnologie wenig bis gar nichts zu tun haben, eine wichtige Rolle gespielt. Das kann man gut an der Arbeit der Kommission des britischen Oberhauses ablesen, die 1999 ebenfalls das Abgehen von Antibiotikaresistenzgenen als Markern empfohlen hat. Die zugrunde liegenden Probleme resultieren allerdings aus der Verwendung von Antibiotika in der fabrikmäßigen Tierhaltung (Rinder, Schweine, Geflügel) als Mastbeschleunigungsmittel und den Tierhaltungsbedingungen.

16) $\mathrm{Zu}$ den Schwierigkeiten der Entwicklung von Alternativen vgl. Koenig 2000; Privalle et al. 2000

17) The Royal Society of Canada 2001; Nature 409: 749 (15. Februar 2001). In der Studie der RSC wird empfohlen: „The Panel recommends that approval of new transgenic organisms for environmental release, and for use as food or feed, should be based on rigorous scientific assessment of their potential for causing harm to the environment or to human health. Such testing should replace the current regulatory reliance on "substantial equivalence" as a decision threshold." (RSC 2001: x).

18) Vgl. bspw. Heasman und Mellentin 2001

19)Damit soll hier keine Parallele von BSE zu trN2 insinuiert werden; wohl soll aber darauf hingewiesen werden, dass bislang nicht praktizierte Verfahren in der Lebensmittelherstellung erhebliche Risiken in sich bergen können, die man weder nach dem Stand der wissenschaftlichen Erkenntnis noch durch übliche Vorsichtsmaßnahmen ausschließen kann. Man kann nur den Versuch unternehmen, durch frühestmögliche und systematische Suche nach Risikoquellen und Vermeidungsstrategien die Risiken zu minimieren. Klar ist, dass damit die Abwägung, ob Risiken in Kauf genommen werden sollen und wofür, noch gar nicht getroffen ist.
20)So z. B. aus der Umweltpolitik, vgl. dazu Sexton et al.1999; Dale \& English 1999

21) Vgl. Bongert 2000; Sauter, Meyer 2000; Spelsberg et al. 2000

22)Die EU-Kommission versucht zwar, auf der Basis der im April 2001 veröffentlichten modifizierten Freisetzungsrichtlinie 90/220/EWG (nunmehr 2001/18/EC) mit dem Vorschlag COM(2001)425 final eine kohärentere Form der Regulierung von novel food und novel feed vorzulegen. Dabei bleiben aber die getrennten Stufen der Zulassung erhalten.

23) Vgl. bspw. zur deutschen Entwicklung Coenen, Petermann et al. 1999; zu Europa Klüver et al. 2000; Vig, Paschen 2000; zu USA Bimber 1996

24)Zu den erkannten Defiziten gehören insbesondere: Asymmetrien zwischen der Herstellung von technologischen Innovationen durch Wissenschaften und Industrien und einer gesellschaftlichen Verständigung über Richtung, Ausmaß und Grenzen; ähnliche Asymmetrien zwischen dem Innovationskomplex und der öffentlichen Politik; der oftmals hermetische Charakter von Expertenstrukturen sowohl in den Wissenschaften wie in hybriden Beratungsgremien mit hohem und decisivem Einfluss; die Abwesenheit parlamentarischer Beratung und Mitbestimmung in Fragen der Lancierung von Forschungsprogrammen, insbesondere in den deutschen Ländern und im Bund, etwas geringer im Europaparlament nach langem und intensivem Kampf; eine zu dem erstgenannten Punkt korrespondierende Schieflage in der Forschungsförderung, weil Implikationsuntersuchungen nicht als sinnvoller Bestandteil von innovationsgerichteter Forschung verstanden wird.

25)Ein solcher normativer Anspruch an die Gestaltungsfähigkeit und -bedürftigkeit technologischer Innovationen stimmt nicht überein mit den landläufigen Vorstellungen von Innovationen als Produkten des wissenschaftlich-technischkommerziell verstandenen „Innovationssystems“. Hier wird vielmehr davon ausgegangen, dass technologische Innovationen den gleichen politischen und auch moralischen Gestaltungsimperativen folgen sollten wie andere Gestaltungen gesellschaftlicher Verhältnisse, vgl. dazu Meyer-Abich 1988, 1997; Sclove 1995; Korten 1998; Monbiot 2000

26) Sicherlich tragen naturwissenschaftliche Erkenntnisse bei zu der Zielangabe „functional food", aber die Intention, derartige Nahrungsmittel zu entwickeln und zu propagieren, ist keine wissenschaftliche, sondern eine gesellschaftspolitische Handlung. Es ist wichtig, diese Tatsache nicht zu übersehen oder zu verwischen. 
27)S. z. B. Food Science Hamburg-Kiel 2001: 2f.; ausführlicher dazu aus einer ebenfalls positiv voreingenommenen Position Hüsing et al. 1999 und, in weiten Teilen inhaltsidentisch, Menrad et al. 2000

28)Oltersdorf \& Ecke 2000: 124

29) Vgl. Hüsing et al. 1999; Menrad et al. 2000 für die Schweiz; für Großbritannien vgl. House of Commons 1999

30)Potrykus \& Beyer 1999; vgl. zum Hintergrund der Finanzierungsprobleme der Forschungen Normile 1999

31) Vgl. hierzu Süddeutsche Zeitung v. 6.3.2001, V2 17

\section{Literatur}

Albrecht, S., 1995: Wie politisch ist die politische Regulierung von technologischen Innovationen? Zum Beispiel Biotechnologie. In: Martinsen, Simonis, S. 137-151

Albrecht, S., 1997: TA zur Biotechnik. So what? In: Martinsen (Hrsg.), 169-187

Ammann, K. et al., 1999: Methods for Risk Assessment of Transgenic Plants. III. Ecological risks and prospects of transgenic plants, where do we go from here? Basel: Birkhäuser

Bimber, B., 1996: The Politics of Expertise in Congress. The Rise and Fall of the Office of Technology Assessment, Albany: The State University of New York Press

Bongert, E., 2000: Regelungen zur Freisetzung, zum Inverkehrbringen und zur Sortenzulassung transgener Nutzpflanzen in der EU. Husum (Gutachten für das Büro für Technikfolgen-Abschätzung beim Deutschen Bundestag)

Bröchler, S. et al. (Hrsg.), 1999: Handbuch Technikfolgenabschätzung, 3 Bde., Berlin: edition sigma

BUND; Misereor (Hrsg.), 1996: Zukunftsfähiges Deutschland. Basel: Birkhäuser

Dale, V.H.; English, M.G. (eds.), 1999: Tools to Aid Environmental Decision Making. New York: Springer

Eurobarometer, 2001: 55.2: Europeans, science and technology, o. O. (Brussels), December

Fairbairn, C. et al., 2000: The Biosafety of Genetically Modified Organisms (Proc. 6th Int. Symp.). Saskatoon: Univ. Ext. Press

Food Science Hamburg-Kiel, 2001: Lipide und Fettsäuren für Lebensmittel mit besonderem gesundheitlichem Wert. Biotechnologische Herstellung, Einsatzmöglichkeiten und gesundheitliche Bewertung, Antrag der Region Hamburg-Kiel zur BMBF-Förderaktivität „BioProfile“, Hamburg
Heasman, M.; Mellentin, J., 2001: The Functional foods Revolution. Healthy People, Healthy Profits? London: Earthscan

Hobbelink, H., 1989: Bio-Industrie gegen die Hungernden. Die Gen-Multis und die Lebens-Mittel der Dritten Welt. Reinbek: Rowohlt

Hobbelink, H., 1991: Biotechnology and the Future of World Agriculture. The Fourth Resource, London

Hohmeyer, O. et al., 1993: Gesetzliche Regelungen der Gentechnik im Ausland und praktische Erfahrungen mit ihrem Vollzug. Karlsruhe: FhG ISI (Gutachten im Auftrag des Büros für Technikfolgen-Abschätzung beim Deutschen Bundestag)

House of Commons Library Research Paper, 1999: Genetically modified crops and food, No. 99/38, London, www.parliament.uk

Hüsing, B. et al., 1999: Functional food - Funktionelle Lebensmittel. Berlin (Gutachten für das Büro für Technikfolgen-Abschätzung beim Deutschen Bundestag)

Jansen, D., 2000: Gesellschaftliche Selbstorganisation durch Technikdiskurse? In: Werle, Schimank, S. 183-207

Kjellsson, G.; Simonsen, V., 1994: Methods for Risk Assessment of Transgenic Plants I. Competition, Establishment and Ecosystem Effects. Basel: Birkhäuser

Kjellsson, G. et al. (ed.), 1997: Methods for Risk Assessment of Transgenic Plants II. Pollination, Gene-Transfer and Population Impacts. Basel: Birkhäuser

Klüver, L. et al., 2000: European Participatory Technology Assessment. Participatory Methods in Technology Assessment and Technology Decision Making. Copenhagen: The Danish Board of Technology

Koenig, A., 2000: Development and biosafety aspects of transgene excision methods. In: Fairbairn et al., pp. $155-170$

Korten, D., 1998: The Post-Corporate World. Life after Capitalism. San Francisco/West Hartford: Berret-Koehler \& Kumarian Press

Lappé, M.; Bailey, B., 1999: Against the Grain. The Genetic Transformation of Global Agriculture. London: Earthscan

Martinsen, R. (Hrsg.), 1997: Politik und Biotechnologie. Baden-Baden: Nomos

Martinsen, R.; Simonis, G. (Hrsg.), 1995: Paradigmenwechsel in der Technologiepolitik? Opladen: Leske + Budrich

Menrad, M. et al., 2000: Functional food. Bern: Schweizerischer Wissenschafts- und Technologierat (Gutachten für das Zentrum für TechnologiefolgenAbschätzung bei Schweizerischen Wissenschaftsund Technologierat) 
Meyer-Abich, K.M., 1988: Wissenschaft für die Zukunft. Holistisches Denken in ökologischer und gesellschaftlicher Verantwortung. München: C.H. Beck

Meyer-Abich, K.M., 1997: Vom Baum der Erkenntnis zum Baum des Lebens. Ganzheitliches Denken der Natur in Wissenschaft und Wirtschaft. München: C.H. Beck

Normile, D., 1999: Rockefeller to End Network After 15 Years of Success. Science 286, 1468-1469

Monbiot, G., 2000: Captive State. The Corporate Takeover of Britain. London: Macmillan

OECD, 1993: Safety Evaluation of Foods Derived Through modern Biotechnology: Concepts and Principles. Paris

OECD, 1996: Food Safety Evaluation. Paris

OECD, 1999: OECD's Task Force for the Safety of Novel foods and Feeds. Paris

Oltersdorf, U.; Ecke, J., 2000: Entwicklungstendenzen bei Nahrungsmittelnachfrage und ihre Folgen. Berlin (Gutachten für das Büro für TechnikfolgenAbschätzung beim Deutschen Bundestag)

Petermann, T. (Hrsg.), 1991: Technikfolgenabschätzung als Technikforschung und Politikberatung. Frankfurt/New York: Campus

Petermann, T.; Coenen, R. (Hrsg.), 1999: Technikfolgenabschätzung in Deutschland. Bilanz und Perspektiven. Frankfurt a. M.: Campus

Potrykus, I., 1991: Gentransfer auf Getreide: eine Abschätzung und Bewertung. In Arbeitsmaterialien zur TA der modernen Biotechnologie, Nr. 2, Hamburg: Universität Hamburg (zuerst engl. BIO/TECHNOLOGY 7, 269-273 (1990)

Potrykus, I.; Beyer, P., 1999: Science 285, 994

Privalle, L.S. et al., 2000: Phosphomannose isomerase - a novel system for plant selection: Mode of action and safety assessment. In: Fairbairn et al., pp. 171-178

Rögener, W., 2001: Gute Absichten, zweifelhafter Nutzen. Süddeutsche Zeitung Nr. 54, 6.3.2001, S. V $2 / 17$

Royal Society of Canada, 2001: Elements of Precaution: Recommendations for the Regulation of Food Biotechnology in Canada. An Expert Panel Report on the future of Food Biotechnology prepared by the RSC at the request of Health Canada, Canadian Food Inspection Agency and Environment Canada. Ottawa: RSC

Sauter, A.; Meyer, R., 2000: Risikoabschätzung und Nachzulassungs-Monitoring transgener Nutzpflanzen. Berlin: Büro für Technikfolgen-Abschätzung beim Deutschen Bundestag (TAB-Arbeitsberichte Nr. 68)
Schütte, G. et al., 1998: Nutzung der Gentechnik im Agrarsektor der USA. 2 Bde. Berlin: Umweltbundesamt

Schütte, G. et al., 2001: Transgene Nutzpflanzen. Sicherheitsforschung, Risikoabschätzung und Nachzulassungs-Monitoring. Basel: Birkhäuser

Sclove, R.E., 1995: Democracy and Technology. New York: The Guildford Press

Sexton, K. et al. (ed.), 1999: Better Environmental Decisions. Strategies for Governments, Businesses, and Communities. Washington, D.C.: Island Press

Simonis, G.; Droz, R., 1999: Die neue Biotechnologie als Gegenstand der Technikfolgenabschätzung und Technikbewertung in Deutschland. In: Bröchler et al., Bd. 3, 909-933

Spelsberg, G. et al., 2000: Novel-Food-Verordnung und transgene landwirtschaftliche Nutzpflanzen. Aachen/Darmstadt (Gutachten für das Büro für Technikfolgen-Abschätzung beim Deutschen Bundestag)

Tiedje, J.M. et al., 1989: Die gezielte Freisetzung genetisch veränderter Organismen: Ökologische Überlegungen und Empfehlungen. In: Arbeitsmaterialien zur TA der modernen Biotechnologie, Nr. 1, Universität Hamburg (zuerst engl. Ecology 70, No. 2, 1989)

topPlantnord, 2001: Schlüsseltechnologien zur Produktion marktfähiger transgener Pflanzen. Hamburg/Kiel

Vig, N.J.; Paschen, H. (eds.), 2000: Parliaments and Technology. The Development of Technology Assessment in Europe. Albany: State University of New York Press

Werle, R.; Schimank, U. (Hrsg.), 2000: Gesellschaftliche Komplexität und kollektive Handlungsfähigkeit. Frankfurt a. M.: Campus

\section{Kontakt}

Dr. Stephan Albrecht

Universität Hamburg

Forschungsschwerpunkt Biotechnik, Gesellschaft und Umwelt (BIOGUM)

Ohnhorststraße 18, 22609 Hamburg

Tel.: +49 (0) 40 / 42816 - 505/- 506

Fax: +49 (0) 40 / 42816 - 527

E-mail: alwold5@aol.com 